\title{
Astragaloside Iv Suppresses Histamine-induced Inflammatory Factors and MUC5AC Overproduction in Nasal Epithelial Cells via Regulation of Inflammation-related Genes
}

Jie Guo ( $\square$ guojie00801@163.com )

Luoyang Central Hospital: Luoyang Central Hospital Affiliated to Zhengzhou University https://orcid.org/0000-0002-1903-6789

\section{Shuai Xu}

Luoyang Central Hospital: Luoyang Central Hospital Affiliated to Zhengzhou University

\section{Research Article}

Keywords: antihistamine, nasal mucosa, allergic rhinitis, MUC5AC, inflammatory cytokine, RNA-SEQ

Posted Date: May 18th, 2021

DOl: https://doi.org/10.21203/rs.3.rs-520631/v1

License: (c) (1) This work is licensed under a Creative Commons Attribution 4.0 International License. Read Full License 


\section{Abstract}

Allergic rhinitis (AR) is a symptomatic allergic disease that leads to severe inflammation. Astragaloside IV (AS-IV) is a primary active component of Astragalus membranaceus and exerts immune-regulation and anti-inflammatory effects. However, the pharmacological effect of AS-IV in the nasal epithelial cells (NECs) has not been reported. The present study aimed to assess the effect of AS-IV on inflammatory cytokines and mucin 5 subtype AC (MUC5AC) overproduction in histamine (His)-stimulated NECs and its underlying mechanism. NECs were stimulated with or without His for $24 \mathrm{~h}$ in the absence or presence of AS-IV. The levels of inflammatory cytokines including IL-6, IL-8, MCP-1, IL-1 colony-stimulating factor (GM-CSF), eotaxin, and MUC5AC were assayed. Our findings indicated that ASIV inhibited His-evoked release and expression of inflammatory cytokines and MUC5AC in NECs. RNASEQ analyses indicated the significant changes in expression levels involved in inflammation genes upon treatment of His-induced NECs with AS-IV. Our findings indicated that AS-IV inhibited His-evoked inflammatory cytokines secretion and MUC5AC overproduction in NECs, which were partly mediated by regulation of inflammation-related genes. AS-IV might be a therapeutic agent for the treatment of AR.

\section{Introduction}

Allergic rhinitis (AR) is a common chronic allergic respiratory disease among children and adults, which occurs in nasal mucosa by the overreaction of allergens [1, 2]. According to epidemiological research, the prevalence of $A R$, which affects 500 million people worldwide, is increasing at an alarming rate [3]. Although AR is not fatal, patients with AR continuously suffer from symptoms, including nasal overflow, rhinorrhea, respiratory obstruction, nasal itching, sneezing, and nasal congestion [4]. Besides, AR patients may also be affected by mood disorders, sleep disorders, and deterioration in social relationships [5]. The symptoms of AR are induced by the secretion of histamine (His), pro-inflammatory cytokines, and allergic, which could induce secretion of tears, and evoke vascular dilation [6]. The airway epithelium is the first line of host defense. Increasing evidence indicated that nasal epithelial cells (NECs) play a vital role in the etiopathogenesis of AR [7]. It has been demonstrated that chronic inflammation of NECs is involved in the pathogenesis of AR [8]. Pro-inflammatory cytokines including interleukin (IL)-6 and IL-8 are highly upregulated in NECs after the stimulation of Dermatophagoides pteronyssinus 1 [7]. Moreover, the inhibition of inflammatory cytokines secretion contributes to suppressing allergic responses in AR [9]. Mucins are highly glycosylated macromolecules secreted by goblet cells in epithelial tissues. However, the overproduction of mucins is a pathognomonic characteristic in chronic airway diseases, which results in the dysfunction of mucociliary in the airways [10]. Mucin 5 Subtype AC (MUC5AC) is considered to be the important mucin in the airway, and the secretion and expression of MUC5AC are increased in nasal allergic inflammation [11]. Therefore, targeting the inflammatory cytokines and MUC5AC might be a potential therapeutic strategy in the treatment of AR. Currently, there are lots of agents that are used for the treatment of $A R$, including immunotherapy, leukotriene receptor antagonists, antihistamines, and nasal steroids. However, those drugs would cause a frequency of side effects, and most patients stop using those agents. Therefore, innovative, safe, and effective therapeutic strategies are still urgently 
needed for AR patients. In recent years, more and more research has focused on the development of natural products derived from medicinal plants for the management of allergic respiratory disease [1214].

Astragalus membranaceus is an herb, which has long been used in China for the treatment of numerous diseases, including kidney diseases, skin diseases, hepatitis, allergic rhinitis, and cardiovascular diseases $[15,16]$. Astragaloside IV (As-IV) is the primary bioactive constituent derived from the Astragalus membranaceus [17]. Accumulated researches indicated that As-IV has diverse pharmacologic activities, such as anti-inflammatory, immunomodulatory, antiviral, and antiapoptosis effects [18]. It has been reported that As-IV could alleviate airway inflammation and airway hyperresponsiveness in an OVAevoked asthma model [19]. Besides, As-IV improved pulmonary function via suppressing the secretion of IL-6 and TNF-a in chronic hypoxia-induced pulmonary hypertension model [20].

However, to the best of our knowledge, the effects of AS-IV on inflammatory cytokines, MUC5AC secretion, and gene expression in NECs have not been reported. Therefore, the present study aimed to investigate the effect of AS-IV on His-evoked inflammatory response and MUC5AC overproduction in NECs.

\section{Materials And Methods}

\section{NECs cultures}

NECs were purchased from Jennio Biotech Co., Ltd. (Guangzhou, China) and maintained in a humidified chamber with $5 \% \mathrm{CO}_{2} / 95 \%$ air at $37{ }^{\circ} \mathrm{C}$. NECs were incubated in BEGM medium (Lonza, Walkersville, Md., USA) contained with $100 \mathrm{U} / \mathrm{ml}$ penicillin (Sigma-Aldrich, MO, USA), $100 \mu \mathrm{g} / \mathrm{ml}$ streptomycin (SigmaAldrich, MO, USA), and 10\% fetal bovine serum (Sigma-Aldrich, MO, USA).

\section{Cell viability measurement}

3-[4,5-dimethylthiazol-2-yl]-2,5 diphenyl tetrazolium bromide (MTT) method was performed to evaluate the cell viability. Briefly, NECs were seeded into 96 -well plates $\left(5 \times 10^{3}\right.$ cells/well). After $24 \mathrm{~h}$ of preincubation, NECs were cultured with different concentrations of His $(0,0.025,0.05$, and $0.1 \mu \mathrm{M}$; SigmaAldrich, MO, USA) or AS-IV $(0,20,40,60$, and $80 \mu \mathrm{M} ;>98 \%$ purity; Aladdin, Shanghai, China). After 24 and $48 \mathrm{~h}$ of incubation, $20 \mu \mathrm{L}$ of MTT solution (Sigma-Aldrich, MO, USA) was transferred to each well and cultured at $37^{\circ} \mathrm{C}$ for another $4 \mathrm{~h}$. Then, the medium was discarded and the generated formazan crystal was dissolved in $200 \mu \mathrm{L}$ of dimethyl sulfoxide (Sigma-Aldrich, MO, USA). Finally, the absorbance was measured in a microplate reader (Bio-Rad, Hercules, CA, USA) at $570 \mathrm{~nm}$.

\section{Treatment of NECs with AS-IV and His stimulation}

AS-IV (Figure 1A, purity $>98 \%$ ) was purchased from Sigma-Aldrich (MO, USA). The NECs were pretreated with AS-IV $(0,20,40$, and $60 \mu \mathrm{M})$ for $30 \mathrm{~min}$. Subsequently, NECs were either unstimulated or stimulated 
with His $(0.1 \mu \mathrm{M})$ for $24 \mathrm{~h}$ in BEGM medium. His and AS-IV were dissolved in $0.1 \%$ dimethyl sulfoxide before the experiment. NECs were dissolved in the same amount of $0.1 \%$ dimethyl sulfoxide in the control group. After $24 \mathrm{~h}$ of incubation, cell pellets were collected for further analysis.

\section{RNA-seq analysis}

The NECs were seeded in cell plates for $24 \mathrm{~h}$. The NECs were pretreated with AS-IV $(60 \mu \mathrm{M})$ for $30 \mathrm{~min}$. Subsequently, NECs were either unstimulated or stimulated with His $(0.1 \mu \mathrm{M})$ for $24 \mathrm{~h}$ in BEGM medium. Total RNA was extracted from NECs using Trizol reagent (Invitrogen, CA, USA). The PCR amplification and sequencing were performed on the GPL16791 Illumina HiSeq 2500 (Homo sapiens). Fold change $\geq 2$ and $p<0.05$ were used as the criteria for the differentially expressed genes (DEGs), and enriched via KEGG analysis.

\section{Measurement of inflammatory cytokines releasing from NECs cells}

NECs cell supernatant was collected by centrifugation at $4,000 \mathrm{~g}$ for 5 min at $4{ }^{\circ} \mathrm{C}$ after $24 \mathrm{~h}$ of cotreatment for inflammatory cytokines measurement. The levels of IL-6, IL-8, MCP-1, IL-1 $\beta$, GM-CSF, and eotaxin in cell culture supernatant were detected by ELISA using commercially available ELISA kits (R\&D Systems, MN, USA) and conducted according to the manufacturer's protocols.

\section{Assay of MUC5AC mucin}

NECs cell supernatant was obtained by centrifugation at $4,000 \mathrm{~g}$ for 5 min at $4{ }^{\circ} \mathrm{C}$ after $24 \mathrm{~h}$ of cotreatment for MUC5AC assay. MUC5AC protein released from cell culture supernatant was detected using Human MUC5AC ELISA kits (NeoMarkers, CA, USA) and performed according to the manufacturer's protocols.

RNA extraction and real-time quantitative reverse transcription-PCR (qRT-PCR)

Total RNA from NECs was extracted using Trizol reagent (Invitrogen, CA, USA) and purified by the RNeasy kit (Qiagen Inc, CA, USA) according to the manufacturer's instructions. Then, reverse-transcription of total RNA was performed by SuperScript III Reverse Transcriptase (Invitrogen, CA, USA). Then, the qRT-PCR was implemented on the ABI Prism 7300 Detection System (Applied Biosystems, CA, USA) by the SYBR Green qPCR Super Mix-UDG kit (Invitrogen, CA, USA). Glyceraldehyde-3-phosphate dehydrogenase (GAPDH) was used for internal calibration. The comparative $2^{-\Delta \Delta C t}$ method was used to calculate the relative mRNA expression. Primer sequences were displayed in Table 1.

\section{Statistical Analysis}

All results were shown as mean \pm SD. Data analysis was carried out using statistical software (GraphPad Software, Inc., La Jolla, CA, USA). The statistical significance of differences among experimental groups was evaluated using one-way ANOVA followed by Dunnett's post hoc test. A value of $P<0.05$ was considered statistical significance. 


\section{Results}

\section{NECs cell viability measurement}

In our study, an MTT assay was carried out to exclude the possibility that the impact of His or AS-IV on cell viability of NECs. As shown in Figure 1B-D, after treatment with different concentrations of His (0-0.1 $\mu \mathrm{M})$ for 24 , and $48 \mathrm{~h}$, or AS-IV $(0-80 \mu \mathrm{M})$ for 24 , and $48 \mathrm{~h}$ for 24 , and $48 \mathrm{~h}$, no significant differences were observed in NECs cell viability. Our findings indicated that the appointed concentrations of His, and AS-IV were non-toxic effects on the cell viability of NECs.

\section{AS-IV inhibited Hisevoked inflammatory cytokine production in NECs}

Previous reports have shown that pro-inflammatory cytokines, including IL-6, IL-8, MCP-1, and IL-1 $\beta$ are up-regulated and play a vital role in AR [21, 22]. Therefore, the pro-inflammatory cytokines were measured in NECs to investigate the effect of AS-IV on the inflammatory response. As shown in Figure 2A-D and Figure 3 , the secretion of IL-6, IL-8, MCP-1, and IL-1 $\beta$, as well as mRNA expression in NECs supernatant were increased after His induction, and pre-treatment with AS-IV $(20,40$, and $60 \mu \mathrm{M})$ inhibited the production of IL-6, IL-8, MCP-1, and IL-1 $\beta$ evoked by His stimulation, indicating that AS-IV suppressed Hisevoked inflammation response in NECs.

\section{AS-IV suppressed His-evoked GM-CSF and eotaxin secretion in NECs}

As shown in Figure 2E-F, the secretion of GM-CSF and eotaxin in NECs supernatant were increased after His induction, and pre-treatment with AS-IV $(20,40$, and $60 \mu \mathrm{M})$ inhibited the secretion of GM-CSF, and eotaxin evoked by His stimulation.

\section{AS-IV suppressed His-evoked MUC5AC secretion in NECS}

As shown in Figure 3, the secretion of MUC5AC in NECs supernatant were increased after His induction, and pre-treatment with AS-IV $(20,40$, and $60 \mu \mathrm{M})$ inhibited the secretion of MUC5AC evoked by His stimulation, indicating that AS-IV suppressed His-evoked MUC5AC secretion in NECs.

\section{Differentially expressed genes (DEGs) profiling by RNA-Seq}

As shown in Figure 4, RNA-SEQ analysis was carried out using His-induced NECs treated with AS-IV. A total of 781 mRNAs with statistically significant differences were screened out in the His group relative to the control group (Figure 4A): there were 400 downregulated genes (green dots) and 381 upregulated genes (red dots). A total of 1215 mRNAs with statistically significant differences were screened out in the His group relative to the His+AS-IV group (Figure 4B): there were 636 downregulated genes (green dots) and 579 upregulated genes (red dots). A total of 1084 mRNAs with statistically significant differences were screened out in the control group relative to the His+AS-IV group (Figure 4C): there were 551 downregulated genes (green dots) and 533 upregulated genes (red dots). Besides, as shown in Figure 5A, heat map indicating the top $25 \mathrm{DEGs}$, and most of these responsible for inflammatory responses and 
inflammatory cytokines. The expression levels of inflammation-related genes, such as IL-18, p65, CCL18, IL-1B, TNF, etc., were up-regulated by His, and were down-regulated after AS-IV treatment. Moreover, Kyoto Encyclopedia of Genes and Genomes (KEGG) analysis (Figure 5B) indicated that genes related to inflammatory pathways, such as cytokine-cytokine receptor interaction, chemokine signaling pathway, and NF-kappa B signaling pathway (Figure 6). These results indicated that the overexpression of inflammation-related singling pathways evoked by His was down-regulated by AS-IV.

AS-IV suppressed the His-induced overexpression of inflammation-related genes

As shown in Figure 7, inflammation-related genes were randomly selected to verify the RNA-SEQ data by qRT-PCR. Our findings showed that the expression of CXCL11, CXCL2, MUC5AC, CCL3, TNF, IL-1B, IL-18, and NF-KB were significantly increased in the His group, and down-regulated by AS-IV. These findings are consistent with the results of transcriptome sequencing, showing that the RNA-SEQ results are reliable, and further demonstrating that AS-IV can inhibit the inflammation-related gene overexpression induced by His.

Suppression of NF-KB signaling pathway inhibited His-evoked secretion and expression of inflammatory cytokines in NECs

Moreover, we also investigated the role of the NF-kB pathway in His-evoked inflammatory cytokines secretion and expression in NECs. As shown in Figure 8, our findings showed that NF-KB inhibitors (NF$\mathrm{KBi}$ ) treatment alleviated His-evoked secretion and expression of inflammatory cytokines in NECs, indicating that AS-IV alleviated His-evoked inflammatory response partly via suppression of NF-KB signaling pathway in NECs.

\section{Discussion}

AR is a type of nasal inflammation induced by inhaled allergens [23]. His is an important inflammatory mediator in the pathogenesis of allergic inflammation, which has been reported to elicit an increase in the secretion of pro-inflammatory mediators and over-expressions of adhesion molecules [24]. Therefore, His is usually used for the establishment of the AR model in vitro. In our research, NECs were stimulated with His. Our findings demonstrated that His-evoked the production and expression of pro-inflammatory mediators (IL-6, IL-8, MCP-1, IL-1 $\beta$, GM-CSF, and eotaxin), and MUC5AC, showing that an AR model was successfully established in vitro.

As-IV is the primary bioactive compound derived from the Astragalus membranaceus, has multiple pharmacological activities. It has been reported that AS-IV attenuates cognitive impairments partly through its anti-inflammatory activities via the suppression of inflammasome overactivation and the TLR4 signaling pathway [25]. And AS-IV protects against lipopolysaccharide-evoked injure by regulating miR-203/MyD88 [26]. In another research, AS-IV attenuates the symptoms of OVA-induced RA via regulating the expression levels of GATA-3, Foxp3, and T-bet [27]. However, no report had focused on the pharmacological effects of AS-IV on AR in His-induced NECs and its potential mechanism has not been 
clarified. In the present research, for the first time, our results indicated that AS-IV inhibited His-evoked secretion and expression of pro-inflammatory cytokines, and MUC5AC, implying that AS-IV attenuated inflammatory response and MUC5AC overexpression in NECs.

AR is characterized by more and more inflammatory factors in lesions, which result in itching, rhinorrhea, and sneezing [28]. It has been reported that activated mast cells generate chemokines, growth factors, cytokines, and mediators that result in the progression of AR [29]. Pro-inflammatory cytokines, such as IL6, IL-8, MCP-1, and IL-1 $\beta$, play an important role in allergen-caused AR and these mediators could be produced by inflammatory cells [29]. GM-CSF and eotaxin are known as pro-inflammatory factors in nasal inflammation that are generated and secreted by fibroblasts, infiltrating leukocytes, and airway epithelial cells in response to inflammatory mediators and allergens [30]. Previous reports have reported that the secretion of IL-6, IL-8, GM-CSF, and eotaxin were significantly increased in vitro AR model by stimulating NECs with inflammatory mediators [31, 32]. In accordance with these findings, in the present study, we found that His increased the levels of IL-6, IL-8, GM-CSF, and eotaxin in NECs. Moreover, AS-IV suppressed the secretion and expression of these mediators in His-stimulated NECs. It has been reported that MUC5AC is a primary member of mucoprotein and is up-regulated in airway epithelial cells due to the stimulation of the airway mucosa to pollutants, pathogens, and allergens [33, 34]. Besides, MUC5AC secretion and expression were increased in His-stimulated NECs [32]. In accordance with the previous report, in the present study, we observed that His increased the secretion and expression of MUC5AC in NECs. Besides, AS-IV inhibited the secretion and expression of MUC5AC in His-stimulated NECs, indicating that AS-IV might be effective in the treatment of AR disease.

Activation of the NF-KB pathway could cause the up-regulation of inflammation-related genes [35]. Hence, inhibition of the NF-KB signaling pathway is an effective therapy for the treatment of inflammation-linked diseases such as AR. A previous report has indicated that Glycyrrhizin suppressed histamine evoked inflammatory response via inactivating the NF-KB pathway in NECs [36]. In accordance with this report, the suppression of the NF-KB pathway inhibited the secretion of IL-6, IL-8, MCP-1, IL-1 $\beta$, GM-CSF, and eotaxin in the His-stimulated NECs. Therefore, we inferred that AS-IV might be considered as a potential candidate drug for the treatment of AR via inhibition of the NF-KB pathway in vitro. However, our research was carried out in NECs, and animal AR models are needed to further verify.

In conclusion, our results revealed that AS-IV inhibited the secretion and expression of IL-6, IL-8, MCP-1, IL$1 \beta$, and MUC5AC in the His-stimulated NECs. Besides, AS-IV treatment inhibited His-evoked upregulation of inflammation-related genes. NF-KBi attenuated His-evoked secretion and expression of IL-6, IL-8, MCP1 , and IL-1 $\beta$ in the His-stimulated NECs. The findings implied that AS-IV suppressed His-evoked inflammation response and upregulation of MUC5AC via inhibition of inflammation-related genes in vitro.

\section{Declarations}

\section{Ethics approval and consent to participate}


This study was approved by the Ethics Committee of Affiliated Luoyang Central Hospital of Zhengzhou University.

\section{Competing interests}

The authors declare that they have no competing interests.

\section{Authors' Contribution}

Jie Guo participated in the experimental design, paper preparation, and data collection. Shuai Xu participated in the paper review, literature search, and funds collection.

\section{Availability of data and materials}

The datasets generated for this study are available on request to the corresponding author.

\section{Acknowledgements}

Not applicable.

\section{Funding}

Not applicable.

\section{Consent for publication}

Not applicable.

\section{References}

1. Tharpe CA, Kemp SF. Pediatric allergic rhinitis. Immunology and allergy clinics of North America 2015; 35:185-98.

2. Khan DA. Allergic rhinitis and asthma: epidemiology and common pathophysiology. Allergy and asthma proceedings $2014 ; 35: 357-61$.

3. Wheatley LM, Togias A. Clinical practice. Allergic rhinitis. The New England journal of medicine 2015; 372:456-63.

4. Tran NP, Vickery J, Blaiss MS. Management of rhinitis: allergic and non-allergic. Allergy, asthma \& immunology research 2011; 3:148-56.

5. Bousquet J, Neukirch F, Bousquet PJ, Gehano P, Klossek JM, Le Gal M, et al. Severity and impairment of allergic rhinitis in patients consulting in primary care. The Journal of allergy and clinical immunology 2006; 117:158-62.

6. Bahekar PC, Shah JH, Ayer UB, Mandhane SN, Thennati R. Validation of guinea pig model of allergic rhinitis by oral and topical drugs. International immunopharmacology 2008; 8:1540-51. 
7. Shi J, Luo Q, Chen F, Chen D, Xu G, Li H. Induction of IL-6 and IL-8 by house dust mite allergen Der p1 in cultured human nasal epithelial cells is associated with PAR/PI3K/NFkappaB signaling. ORL; journal for oto-rhino-laryngology and its related specialties 2010; 72:256-65.

8. Borish L. Allergic rhinitis: systemic inflammation and implications for management. The Journal of allergy and clinical immunology 2003; 112:1021-31.

9. Lin H, Zheng C, Li J, Yang C, Hu L. Lentiviral shRNA against KCa3.1 inhibits allergic response in allergic rhinitis and suppresses mast cell activity via PI3K/AKT signaling pathway. Scientific reports 2015; 5:13127.

10. Rose MC, Voynow JA. Respiratory tract mucin genes and mucin glycoproteins in health and disease. Physiological reviews 2006; 86:245-78.

11. Shah SA, Ishinaga H, Hou B, Okano M, Takeuchi K. Effects of interleukin-31 on MUC5AC gene expression in nasal allergic inflammation. Pharmacology 2013; 91:158-64.

12. Ryu J, Lee HJ, Park SH, Sikder MA, Kim JO, Hong JH, et al. Effect of Prunetin on TNF-a-Induced MUC5AC Mucin Gene Expression, Production, Degradation of IKB and Translocation of NF-kB p65 in Human Airway Epithelial Cells. Tuberculosis and respiratory diseases 2013; 75:205-9.

13. Liu T, Xu J, Wu Y, Li X, Ding D, Ma D, et al. Beneficial effects of baicalein on a model of allergic rhinitis. Acta pharmaceutica (Zagreb, Croatia) 2020; 70:35-47.

14. $\mathrm{Hu} \mathrm{H}$, Li H. Prunetin inhibits lipopolysaccharide-induced inflammatory cytokine production and MUC5AC expression by inactivating the TLR4/MyD88 pathway in human nasal epithelial cells. Biomedicine \& pharmacotherapy = Biomedecine \& pharmacotherapie 2018; 106:1469-1477.

15. Ren S, Zhang H, Mu Y, Sun M, Liu P. Pharmacological effects of Astragaloside IV: a literature review. Journal of traditional Chinese medicine = Chung i tsa chih ying wen pan 2013; 33:413-6.

16. Guo H, Liu MP. Mechanism of traditional Chinese medicine in the treatment of allergic rhinitis. Chinese medical journal 2013; 126:756-60.

17. Li L, Hou X, Xu R, Liu C, Tu M. Research review on the pharmacological effects of astragaloside IV. Fundamental \& clinical pharmacology 2017; 31:17-36.

18. Zhang J, Wu C, Gao L, Du G, Qin X. Astragaloside IV derived from Astragalus membranaceus: A research review on the pharmacological effects. Advances in pharmacology (San Diego, Calif) 2020; 87:89-112.

19. Yang $X$, Wang $F$. The effect of astragaloside IV on JAK2-STAT6 signalling pathway in mouse model of ovalbumin-induced asthma. Journal of animal physiology and animal nutrition 2019; 103:15781584.

20. Zhang X, Chen J, Xu P, Tian X. Protective effects of astragaloside IV against hypoxic pulmonary hypertension. MedChemComm 2018; 9:1715-1721.

21. Cui XY, Chen X, Yu CJ, Yang J, Lin ZP, Yin M, et al. Increased expression of toll-like receptors 2 and 4 and related cytokines in persistent allergic rhinitis. Otolaryngology-head and neck surgery : official journal of American Academy of Otolaryngology-Head and Neck Surgery 2015; 152:233-8. 
22. Gröger M, Klemens C, Wendt S, Becker S, Canis M, Havel M, et al. Mediators and cytokines in persistent allergic rhinitis and nonallergic rhinitis with eosinophilia syndrome. International archives of allergy and immunology 2012; 159:171-8.

23. Bousquet J, van Cauwenberge P, Aït Khaled N, Bachert C, Baena-Cagnani CE, Bouchard J, et al. Pharmacologic and anti-lgE treatment of allergic rhinitis ARIA update (in collaboration with GA2LEN). Allergy 2006; 61:1086-96.

24. Gelfand EW. Role of histamine in the pathophysiology of asthma: immunomodulatory and antiinflammatory activities of H1-receptor antagonists. The American journal of medicine 2002; 113 Suppl 9A:2s-7s.

25. Li M, Li H, Fang F, Deng X, Ma S. Astragaloside IV attenuates cognitive impairments induced by transient cerebral ischemia and reperfusion in mice via anti-inflammatory mechanisms. Neuroscience letters 2017; 639:114-119.

26. Li D, Li G, Chen Y, Li Y, Zhang J, Gao D, et al. Astragaloside IV protects ATDC5 cells from lipopolysaccharide-caused damage through regulating miR-203/MyD88. Pharmaceutical biology 2020; 58:89-97.

27. Li K, Chen Y, Jiang R, Chen D, Wang H, Xiong W, et al. Protective effects of astragaloside IV against ovalbumin-induced allergic rhinitis are mediated by T-box protein expressed in T cells/GATA-3 and forkhead box protein 3/retinoic acid-related orphan nuclear receptor yt. Molecular medicine reports 2017; 16:1207-1215.

28. Yuan X, Ghosh A, Jie Q, He G, Wu Y. Effects of desloratadine citrate disodium injection on rat models of ovalbumin-induced allergic rhinitis: involvement of T-cell responses modulation. International forum of allergy \& rhinology 2015; 5:1170-6.

29. Galli SJ, Tsai M. IgE and mast cells in allergic disease. Nature medicine 2012; 18:693-704.

30. Matsuwaki Y, Wada K, White T, Moriyama H, Kita H. Alternaria fungus induces the production of GMCSF, interleukin-6 and interleukin-8 and calcium signaling in human airway epithelium through protease-activated receptor 2. International archives of allergy and immunology 2012; 158 Suppl 1:19-29.

31. Wang B, Gao Y, Zheng G, Ren X, Sun B, Zhu K, et al. Platycodin D inhibits interleukin-13-induced the expression of inflammatory cytokines and mucus in nasal epithelial cells. Biomedicine \& pharmacotherapy = Biomedecine \& pharmacotherapie 2016; 84:1108-1112.

32. Sun B, Wang B, Xu M. Esculetin inhibits histamine-induced expression of inflammatory cytokines and mucin in nasal epithelial cells. Clinical and experimental pharmacology \& physiology 2019; 46:821827.

33. Voynow JA, Gendler SJ, Rose MC. Regulation of mucin genes in chronic inflammatory airway diseases. American journal of respiratory cell and molecular biology 2006; 34:661-5.

34. Thai P, Loukoianov A, Wachi S, Wu R. Regulation of airway mucin gene expression. Annual review of physiology 2008; 70:405-29. 
35. Lawrence T. The nuclear factor NF-kappaB pathway in inflammation. Cold Spring Harbor perspectives in biology 2009; 1:a001651.

36. Li H, Guo D, Zhang L, Feng X. Glycyrrhizin attenuates histamine-mediated MUC5AC upregulation, inflammatory cytokine production, and aquaporin 5 downregulation through suppressing the NF-KB pathway in human nasal epithelial cells. Chemico-biological interactions 2018; 285:21-26.

\section{Tables}

Table 1. Primer sequences for quantitative real-time RNA.

\begin{tabular}{|lll|}
\hline Genes & Forward primer & Reverse primer \\
\hline CXCL11 & 5'-ATGAGTGTGAAGGGCATGGC-3' & 5'-TCACTGCTTTTACCCCAGGG-3' \\
\hline CXCL2 & 5'-GCTGCTGCTCCTGCTTCTAGTG-3' & 5'-AGGTGAATTCCTTGCACGGTCTG-3' \\
\hline CCL3 & 5'-CATGGCGCTCTGGAACGAA-3' & 5'-TGCCGTCCATAGGAGAAGCA-3' \\
\hline TNF & 5'-GCACTGAGAGCATGATCCGAGAC-3' & 5'-CGACCAGGAGGAAGGAGAAGAGG-3' \\
\hline IL-1B & 5'-AAGTGATGGCTAACTACGGTGACAAC-3' & 5'-GCTTCTCCACTGCCACGATGAC-3' \\
\hline IL-18 & 5'-GCTTGAATCTAAATTATCAGTC-3' & 5'-GAAGATTCAAATTGCATCTTAT-3' \\
\hline MUC5AC & 5'-CGACAACTACTTCTGCGGTGC-3' & 5'-GCACTCATCCTTCCTGTCGTT-3' \\
\hline NF-KB & 5'-AGCACCAAGACCGAAGCAA-3' & 5'-TCTCCCGTA ACCGCGTAGTC-3' \\
\hline GAPDH & 5'-CAACTTTGGCATTGTGGAAGG-3' & 5'-ACACATTGGGGGTAGGAACAC-3' \\
\hline
\end{tabular}

\section{Figures}
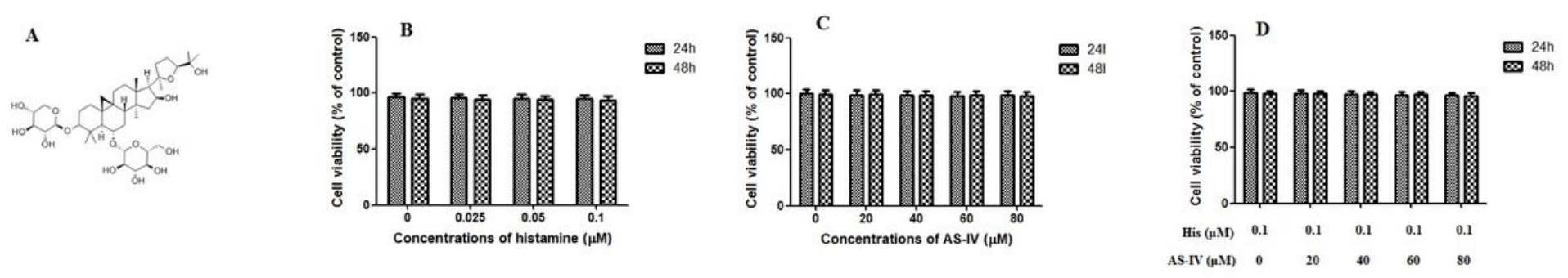

Figure 1 
Effect of histamine, and AS-IV on cell viability of nasal epithelial cells. Chemical structure of AS-IV (A). Effect of histamine on cell viability of nasal epithelial cells (B). Effect of AS-IV on cell viability of nasal epithelial cells (C). Effect of His + AS-IV on cell viability of nasal epithelial cells (D). The results were expressed as the mean \pm SD of three independent experiments.
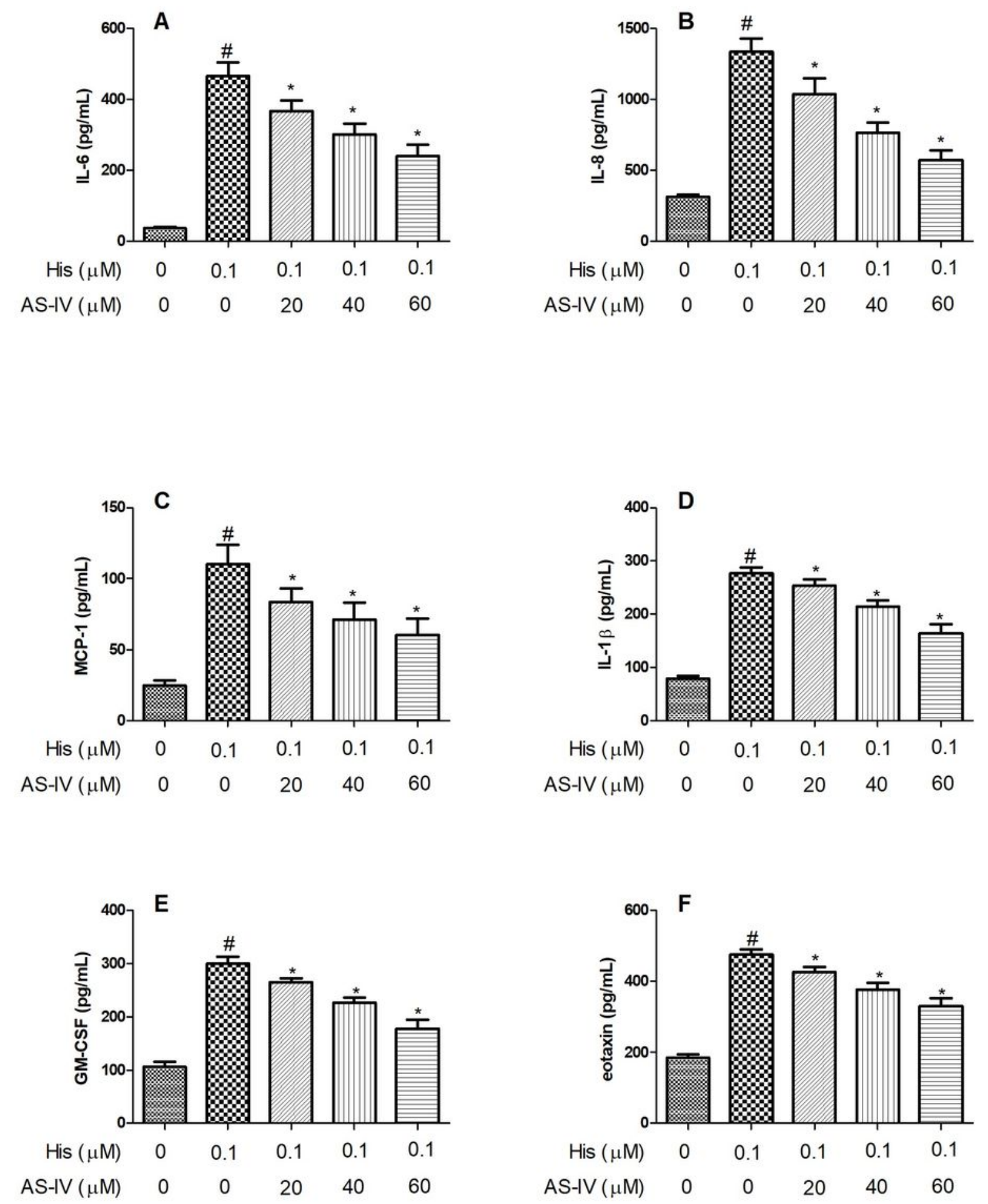

Figure 2 
AS-IV inhibited His-evoked inflammatory cytokine production in nasal epithelial cells. The levels of IL-6 $(A), I L-8(B)$, MCP-1 (C), IL-1 $\beta(D)$, GM-CSF (E), and eotaxin (F) were assayed by ELISA. The results were expressed as the mean \pm SD of three independent experiments. $\# \mathrm{P}<0.01$ vs. control group, ${ }^{*} \mathrm{P}<0.01$ vs. His group. AS-IV inhibited His-evoked inflammatory cytokine overexpression in nasal epithelial cells. The mRNA expression levels of IL-6 (A), IL-8 (B), MCP-1 (C), and IL-1 $\beta$ (D) were assayed by RT-PCR. The results were expressed as the mean \pm SD of three independent experiments. $\# P<0.01$ vs. control group, $* P<$ 0.01 vs. His group.

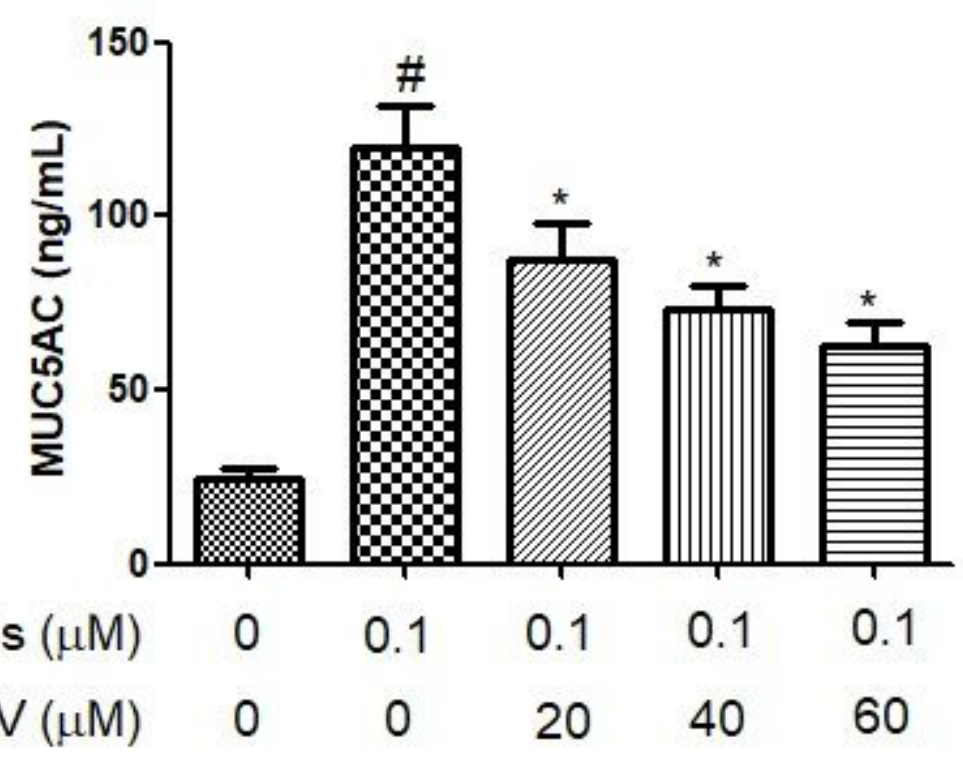

Figure 3

AS-IV suppressed His-evoked MUC5AC overproduction in nasal epithelial cells. The contents of MUC5AC were assayed using ELISA. The results were expressed as the mean \pm SD of three independent experiments. \#P< 0.01 vs. control group, ${ }^{\star} \mathrm{P}<0.01$ vs. His group. 

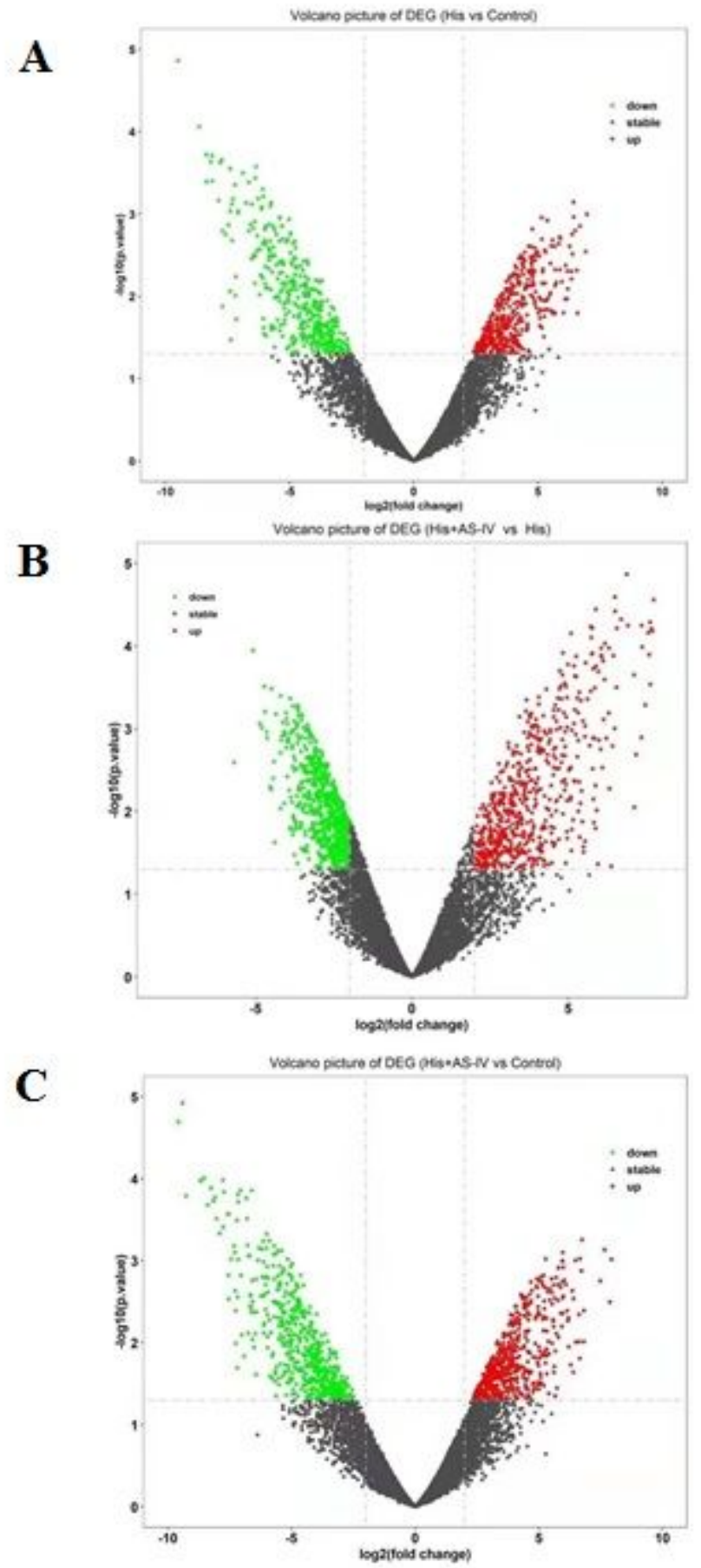

Figure 4

Volcano plot showing DEGs between two groups (A, B, and C). Green, gray, and red dots in the Volcano plot showed significantly downregulated genes, insignificantly DEGs, upregulated genes, respectively. The DEGs with fold change $\geq 2.0$ and $p<0.05$ and are shown. 
A

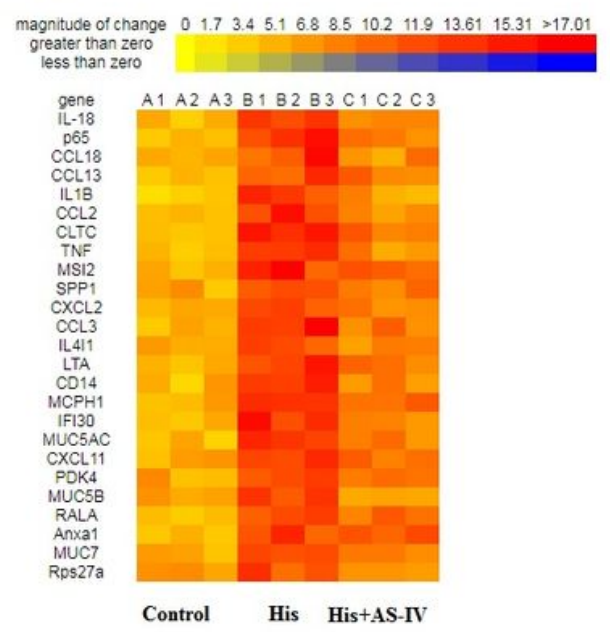

B

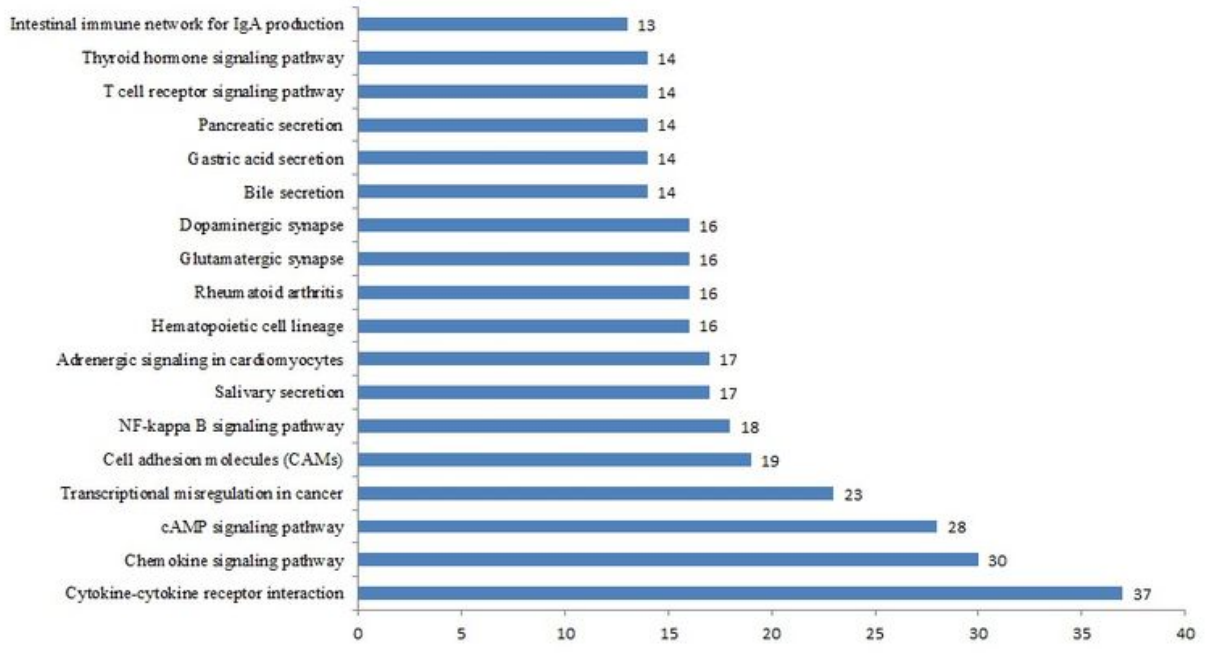

\section{Figure 5}

Heatmap diagram indicating that the common DEGs in the three groups (A). Red and yellow squares in the heatmap showed mRNAs with up-regulated and down-regulated expression levels, respectively. KEGG pathway analyses of the overlapping genes (B). 


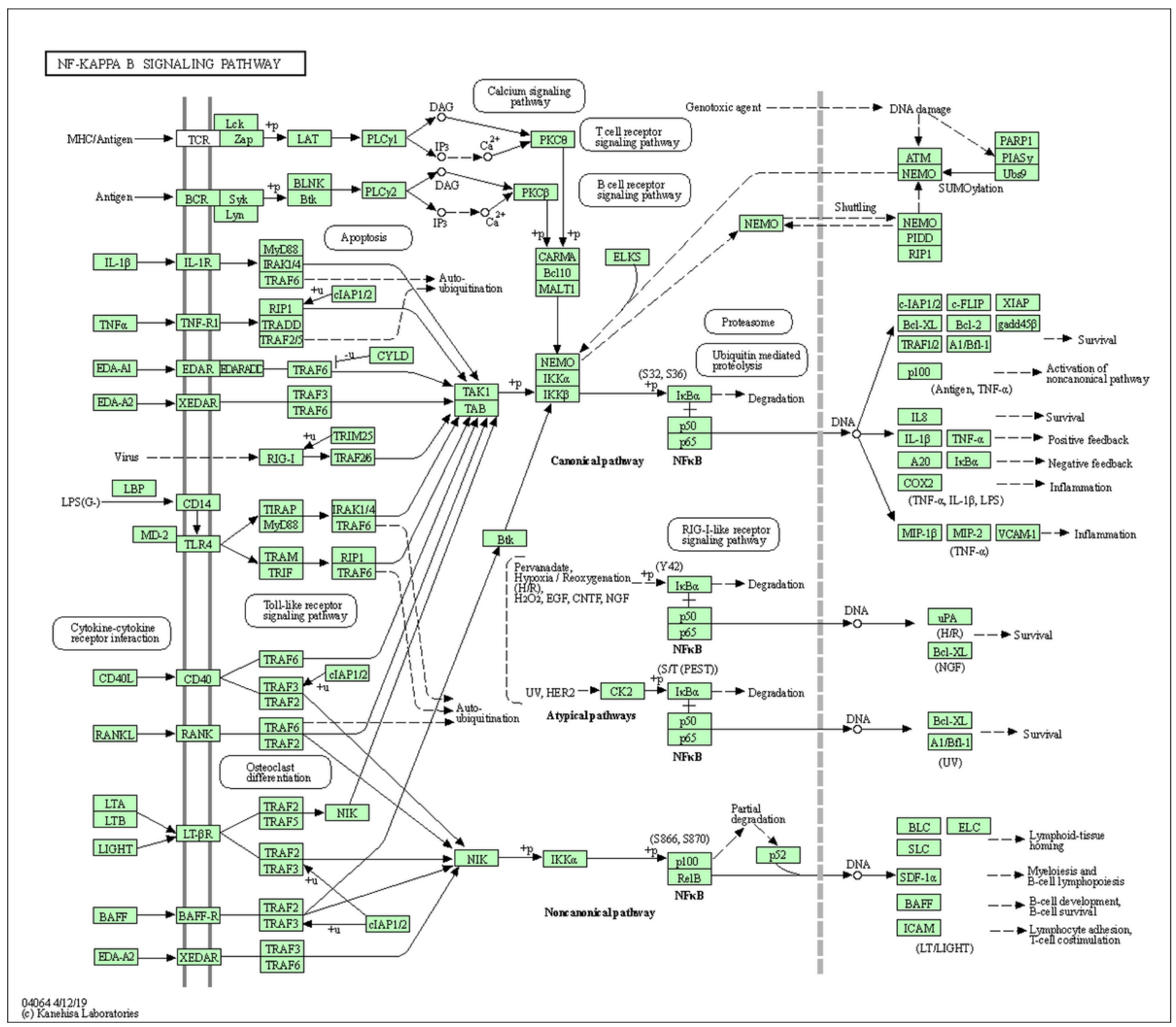

Figure 6

Enrichment pathway of KEGG in NF-KAPPA B signaling pathway. 

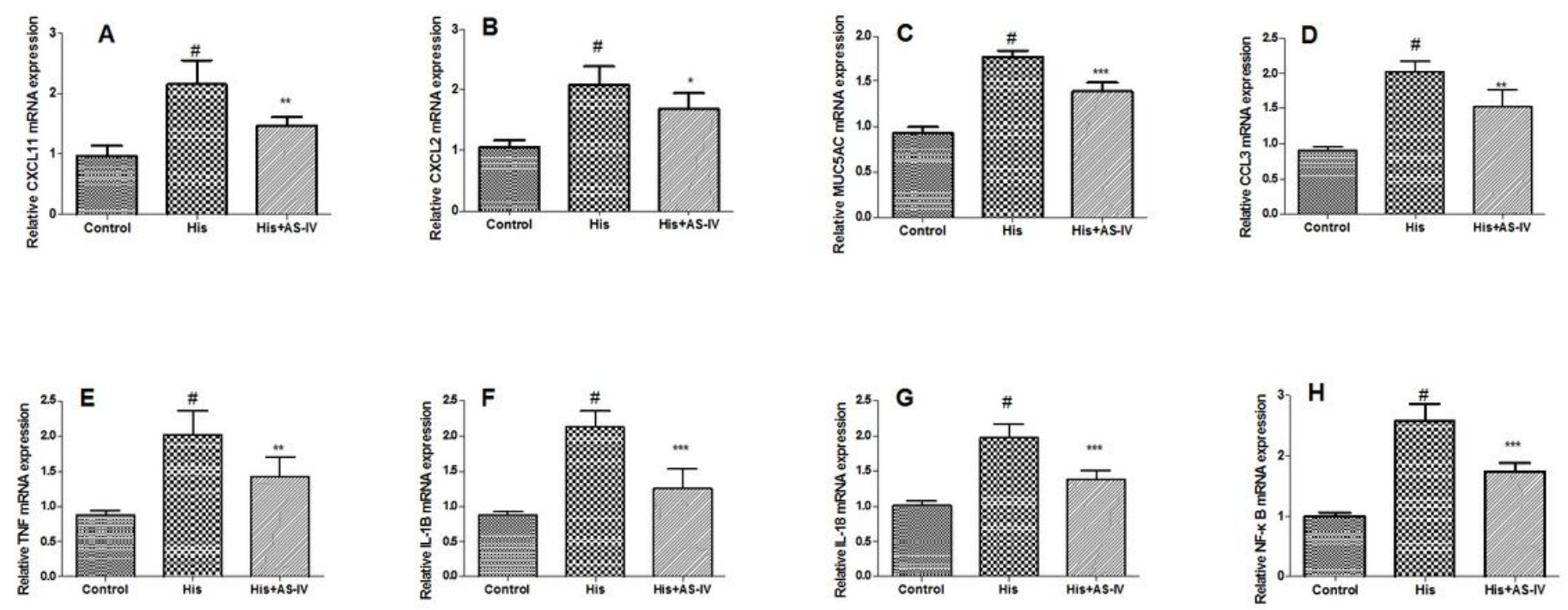

Figure 7

Validation of RNA-SEQ results using quantitative real-time polymerase chain reaction (qRT-PCR) analysis. The results were expressed as the mean $\pm S D$ of three independent experiments. $\# P<0.01 \mathrm{vs}$. control group, ${ }^{\mathrm{P}}<0.01$ vs. His group. 

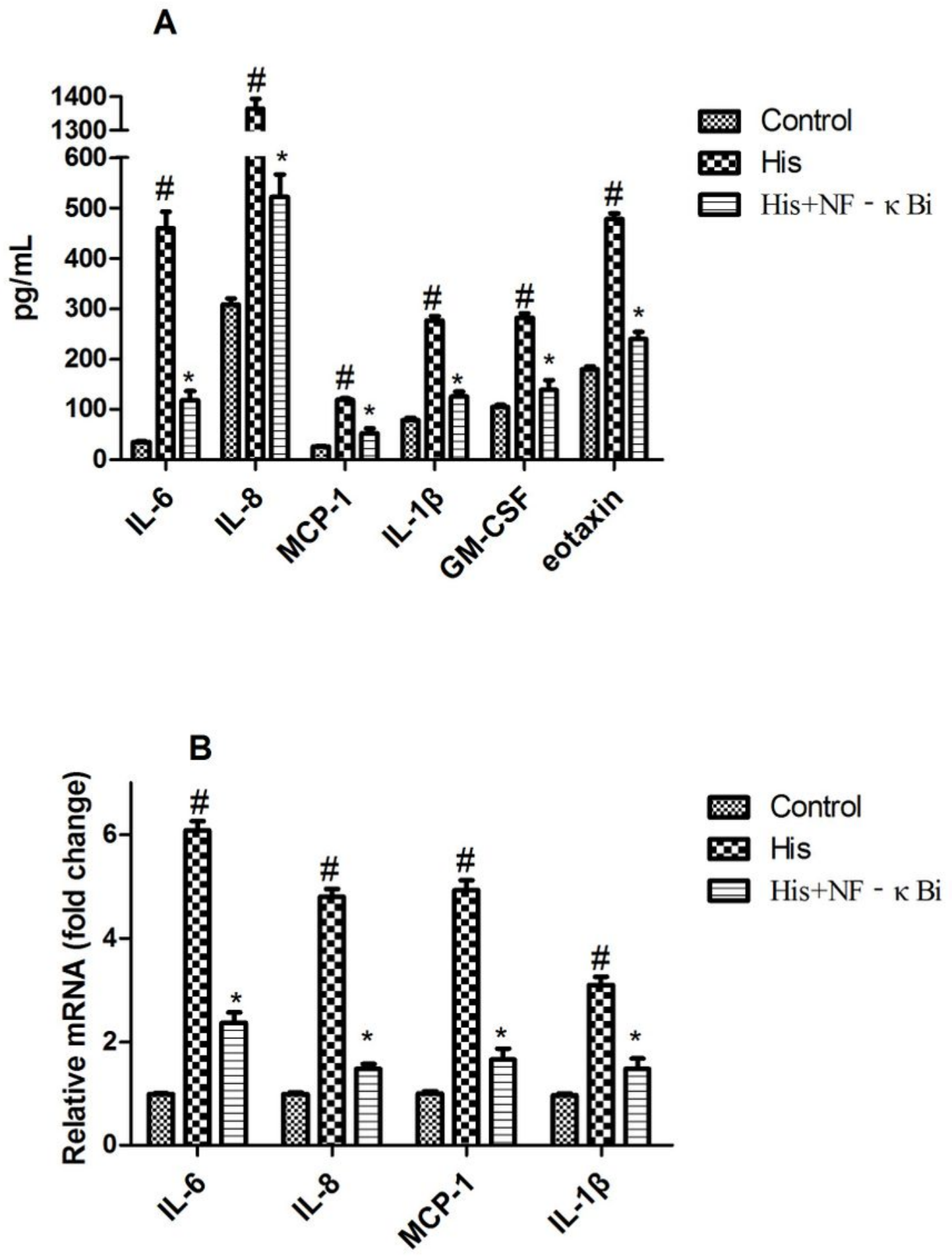

Figure 8

Suppression of the NF-KB signaling pathway decreased His-evoked inflammatory cytokine response in nasal epithelial cells. The effect of NF-KB inhibitor (NF-kBi) on His-evoked secretion of IL-6, IL-8, MCP-1, IL-1 $\beta$, GM-CSF, and eotaxin (A). The effect of NF-kBi on His-evoked mRNA expression of IL-6, IL-8, MCP-1, and IL-1 $\beta(B)$. The results were expressed as the mean \pm SD of three independent experiments. $\# P<0.01$ vs. control group, ${ }^{*} \mathrm{P}<0.01$ vs. His group. 